\title{
Liganded retinoic acid $X$ receptor $\alpha$ represses connexin 43 through a potential retinoic acid response element in the promoter region
}

\author{
Ruoyi Gu', Jun Xu', Yixiang Lin', Jing Zhang ${ }^{1,4}$, Huijun Wang ${ }^{1,2}$, Wei Sheng ${ }^{1,2}$, Duan Ma ${ }^{2,3}$, Xiaojing Ma ${ }^{1,2}$ and Guoying Huang ${ }^{1,2}$
}

\begin{abstract}
INTRODUCTION: Retinoic acid $X$ receptor alpha $(R X R a)$ and Connexin 43 (CX43) both play a crucial role in cardiogenesis. However, little is known about the interplay mechanism between the RXRa and CX43.

METHODS: The activations of retinoic acid response element (RARE) in C $x 43$ were measured by luciferase transfection assay. Electrophoretic mobility shift assay (EMSA) and chromatin immunoprecipitation (ChIP) was performed to prove that $R X R a$ can directly bind to the RARE sequence. Quantitative real-time polymerase chain reaction (qRT-PCR) and western blotting were used to analyze the RXRa and CX43 mRNA level and protein level in cells.
\end{abstract}

RESULTS: In this study, we confirmed the negative association of the gene expression between the RXRa and $C \times 43$ in the cell level. Interestingly, a functional RARE was detected in the region from $-1,426$ to -314 base pairs upstream from the transcriptional start site of $C \times 43$. Moreover, we also prove that $R X R a$ can directly bind to this RARE sequence in vitro and in vivo.

CONCLUSIONS: RXRa negatively regulates the transcription and expression by directly binding to the RARE in the promoter of $C \times 43$. The RARE-like sequence harbored in the $C \times 43$ promoter region may serve as a functional RARE in the retinoic acid (RA) signaling pathway.

Clive ongenital heart disease is the most common birth defect in live neonatal infants, with a prevalence of 8 to 12 per 1,000 live births $(1,2)$. Cardiac development requires a precise and extremely complex series of molecular mechanisms to maintain the proper expression of cardiac transcription factors, and abnormal alterations in these expressions can result in heart defects $(3,4)$. However, the pathogenesis of congenital heart disease is not completely understood.

Connexin 43 (Cx43), also known as GJA1, is a member of the connexin multi-gene family. In the human adult heart, Cx43 forms intercellular communication channels at the plasma membrane to ensure electrical and metabolic coupling between cells $(5,6)$. In addition to its role in adult hearts, $C \times 43$ is also important for normal embryonic heart development. Some investigators have altered the gene dosage of $C x 43$ in the animal model and found pulmonary outflow tract obstruction or conotruncal heart malformation in mice, confirming the importance of $C x 43$ in conotruncal heart development $(7,8)$. However, an alteration in the $C x 43$ protein status inducing heart defects in human has not been confirmed (9). Some studies showed mutations in the $C x 43$ sequence were not commonly associated with human heart malformation (10-12), indicating that the $C x 43$ sequence defect might not be a major contributor to congenital heart disease; the pathogenic mechanism is likely to be a more complex regulatory pathway.

Retinoid acid (RA) is produced by the metabolism of retinaldehyde from retinoids by retinaldehyde dehydrogenase (RALDH) $(13,14)$. RA activates or represses target genes through the activation of two members of the nuclear receptor super family, retinoic acid receptors and retinoid X receptors (RXRs), binding to the retinoic acid response element (RARE) in target genes (14-16). In some cancer studies (17-19), RA has been found to alter the phosphorylation status of the $C \times 43$ protein. Only a few studies have demonstrated the regulation relationship between RA and Cx43 in heart development (20), and the phosphorylation status is not a main cause of heart defects (10). It is very important for us to investigate the mechanism of how RA regulates $C x 43$ in cardiogenesis. Our previous studies indicated that elevated methylation at the retinoid X receptor $\alpha(R X R \alpha)$ promoter may be responsible for downregulating mRNA expression in the right ventricular outflow tract (RVOT) myocardium of patients with TOF (21), while our another observation in TOF patients found that the expression of $C x 43$ increased (22). Thus, we used 9-cis RA (9cRA) to up-regulate endogenous $R X R \alpha(23,24)$, which decreased $C x 43$ expression. We also used $\operatorname{sh} R X R \alpha$ to repress its expression and found that $C x 43$ expression increased. Therefore, we hypothesize that $R X R \alpha$ could regulate $C x 43$ expression, but the precise mechanism remains unknown. We searched further for the $C x 43$ promoter region and detected a RARE-like construction that was located -424 to -407 base pairs upstream of the transcription start site. There

'Children's Hospital of Fudan University, Shanghai, China; ${ }^{2}$ Shanghai Key Laboratory of Birth Defects, Shanghai, China; ${ }^{3}$ Key Laboratory of Molecular Medicine, Ministry of Education, Shanghai Medical College, Fudan University, Shanghai, China; ${ }^{4}$ Present address: Department of Pediatrics, Chengdu Women and Children's Medical Center, Sichuan, China. Correspondence: Guoying Huang (gyhuang@shmu.edu.cn)

Received 18 May 2015; accepted 28 September 2015; advance online publication 20 April 2016. doi:10.1038/pr.2016.47 

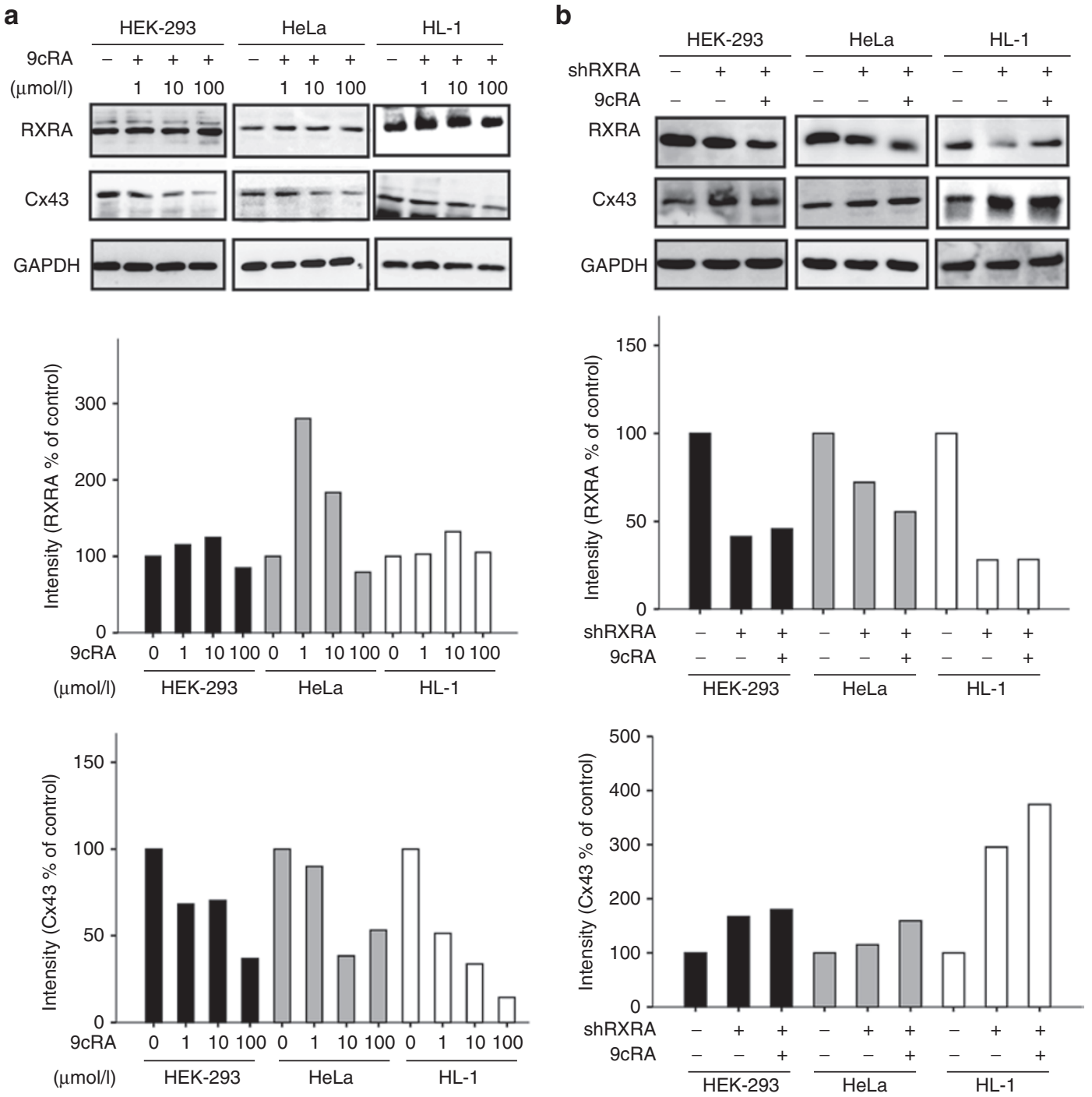

Figure 1. Liganded $R X R \alpha$ had a negative regulation on $C x 43$ protein levels. (a) The cells were treated with 9cRA for $24 \mathrm{~h}$; the proteins were extracted and analyzed by western blotting. The bar graph compares the relative intensity of $C x 43$ or RXR $\alpha$ band normalized to the GAPDH loading control. (b) The cells were transfected with pGreen-shRXR $\alpha$ and treated with 9cRA for $24 \mathrm{~h}$. Proteins were analyzed by western blotting as described in Figure 1 , panel a.

is little evidence of a functional RARE in the $C \times 43$ promoter. It is necessary to investigate whether $R X R \alpha$ could regulate $C x 43$ through this RARE in the $C x 43$ gene.

\section{RESULTS}

\section{9-cis Retinoic Acid Represses Cx43 Gene Expression by Upregulating Endogenous $R X R \alpha$}

Western blotting analyses indicated that the endogenous $R X R \alpha$ expression increased after 9cRA stimulation and that the $C \times 43$ level decreased as the 9cRA concentration increased (Figure 1, panel a). However, Cx43 expression was upregulated after $\operatorname{sh} R X R \alpha$ was used to suppress $R X R \alpha$ and was not influenced by 9cRA (Figure 1, panel b). A quantitative realtime polymerase chain reaction (qRT-PCR) experiment was performed in HEK293 cells that were treated with different concentrations of $9 \mathrm{cRA}$ for $24 \mathrm{~h}$, which showed that the mRNA expression level of $C x 43$ decreased $~ 2.6$-fold $(1 \mu \mathrm{mol} / 1$ 9cRA $)$ and $\sim 3.6$-fold $(10 \mu \mathrm{mol} / 1$ 9cRA $)(P<0.001$; Figure 2, panel a, right). Similar results were observed in HeLa cells and HL- 1 cells. The Cx43 mRNA level decreased $~ 2.1$-fold (1 $\mu \mathrm{mol} / \mathrm{l} 9 \mathrm{cRA})$ and $\sim 2.7$-fold $(10 \mu \mathrm{mol} / 1 \mathrm{9cRA})$ in HeLa cells $(P<0.001$; Figure 2, panel a, right), while in HL-1 cells, this level decreased $\sim 1.7$-fold $(1 \mu \mathrm{mol} / \mathrm{l} 9 \mathrm{cRA})$ and $\sim 1$.4-fold (10 $\mu \mathrm{mol} / 1$ 9cRA) $(P<0.001$; Figure 2, panel a, right). As shown in Figure 2, panel $\mathbf{b}$ (left), $\operatorname{sh} R X R \alpha$ successfully repressed the mRNA level of RXR $\alpha$ in the three cell lines. The Cx43 mRNA level was $\sim 2$-fold higher than that in the control in HEK293 cells $(P<0.05$; Figure 2, panel b, right). In HeLa cells and HL-1 cells, the mRNA level of $C x 43$ increased approximately $\sim 1.5$-fold and $\sim 1.75$ fold $(P<0.05$; Figure 2, panel $\mathbf{b}$, right). Moreover, $C x 43 \mathrm{mRNA}$ also increased in $R X R \alpha$ silenced group when 9cRA existed ( $P<0.05$; Figure 2 , panel $\mathbf{b}$, right).

To confirm whether $R X R \alpha$ is located in the nucleus and could regulate $C \times 43$ expression, we performed an immunofluorescence assay with antibodies against $R X R \alpha$ and $C \times 43$. As shown in Figure 3, the RXR $\alpha$ protein was localized in the nucleus, and 

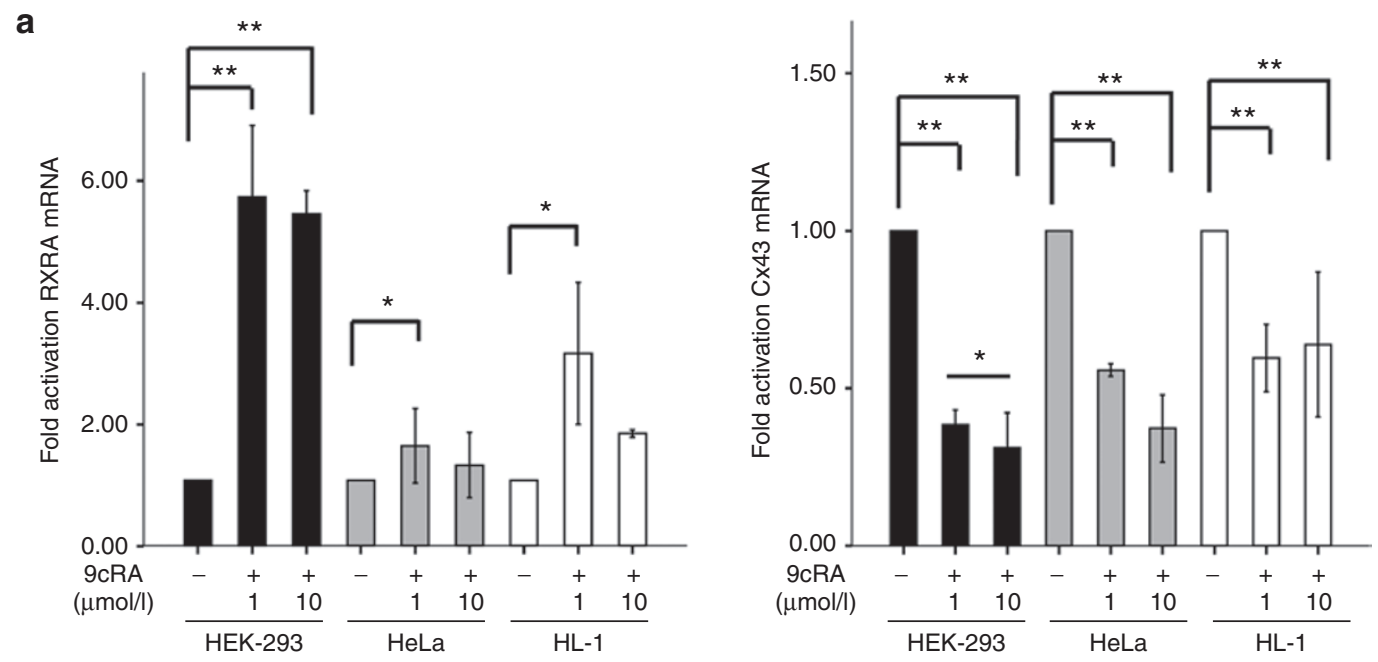

b
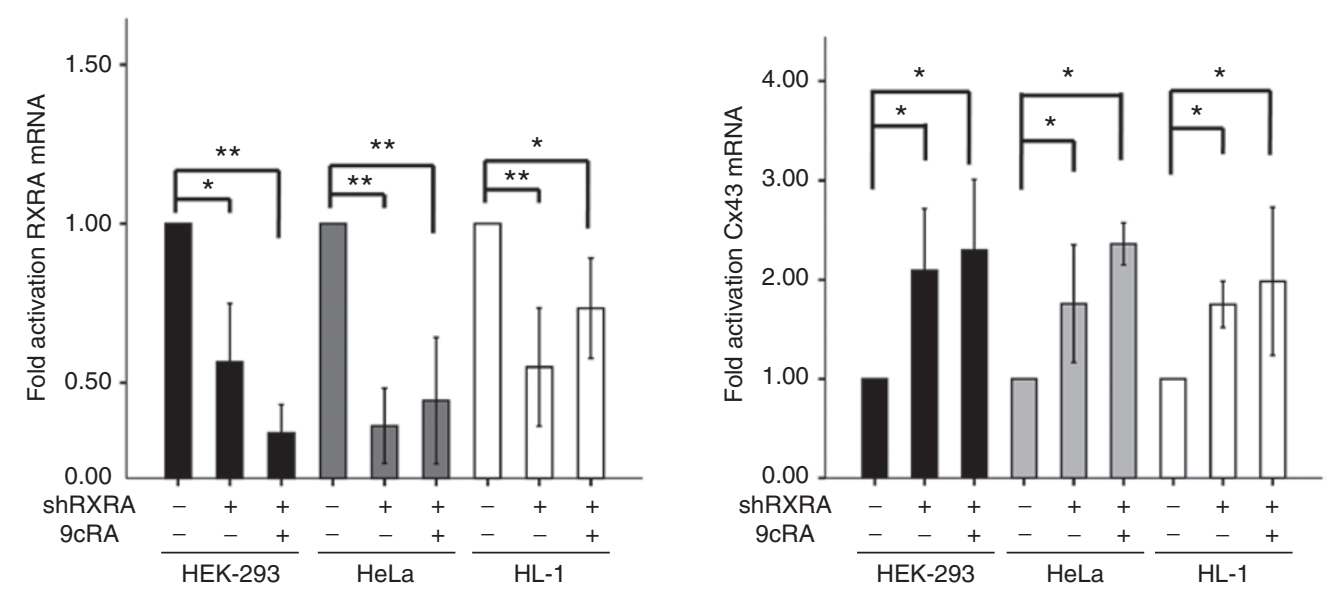

Figure 2. Liganded $R X R \alpha$ had a negative regulation on $C X 43$ mRNA levels. The total RNA from three cells lines was harvested for quantitative real-time polymerase chain reaction analysis. (a) The cells treated with difference concentration of 9cRA. (b) The cells transfected with shRXR $\alpha$ and treated with 10 $\mu \mathrm{mol} / \mathrm{l}$ 9cRA. Bars, SE. ${ }^{*} P<0.05,{ }^{* *} P<0.001$.

Cx43 was located on the cell surface and cytoplasm. Consistent with the western blotting results, the expression of $C x 43$ was higher than that of the control group with 9cRA treatment, and $C \times 43$ increased when $R X R \alpha$ was downregulated.

\section{$R X R \alpha$ Represses the CX43 Promoter via RARE Directly in the Presence of 9cRA}

An analysis of the promoter region of the $C x 43$ gene revealed putative RAREs ranging from -424 to -407 base pairs upstream of the transcription start site (TSS). This RARE in the Cx43 promoter was conserved between human, mouse, dog, and elephant (ENSG00000152661; Figure 4, panel a) (25).

To investigate whether 9cRA could repress Cx43 expression by $R X R \alpha$ binding this putative RARE, we performed a luciferase assay using HEK293, HeLa and HL-1 cells that were cotransfected with the $R X R \alpha$ expression vector and a luciferase vector containing 1426 base pairs of the human $C \times 43$ promoter. In the presence of $9 \mathrm{cRA}$, we observed a significant decrease in the relative luciferase activation of approximately 2.2-fold in HEK293, 1.8-fold in HeLa and 2.4-fold in HL- 1 cells, indicating that this region of the Cx43 promoter might harbor a RARE $(P<0.001$, HEK293; $P<0.001$, HeLa; $P<0.001$, HL-1; Figure 4, panel b, left). Then, a transient cotransfection experiment was performed to examine the effect of mutating this sequence on the promoter activity in the three cell lines. As expected, the luciferase activation was much lower in the mutant RARE (mutRARE) than in the wild-type in the non-RA group $(P<0.001$, HEK293; $P<$ $0.001, \mathrm{HeLa} ; P<0.001$, HL-1; Figure 4, panel c), and luciferase activation showed no difference in the mutRARE group with 9cRA ( $P=0.889$, HEK293; $P=0.932$, HeLa; $P=0.387$, HL-1; Figure 4, panel b, right). Our results showed that mutRARE lost not only the basal activity of the CX43 promoter in the non-RA group but also the inhibitory effect of RARE in the presence of 9cRA (Figure 4, panel b; Figure 4, panel c). To further confirm the function of the putative RARE, we also transfected pGL3-Cx43-Luc or pGL3-mutCx43-Luc to cells in which $R X R \alpha$ were knocked out by pGreen-shRXR $\alpha$. In the pGL3-Cx43-Luc group, the relative luciferase activation increased $\sim 1.50$-fold in HEK293 cells, $\sim 1.59$-fold in HeLa cells and 2.44-fold in HL- 1 cells $(P<0.001$, HEK293; $P<0.001$, HeLa; $P<0.001$, HL-1; Figure 4, panel e, left). The relative 
a

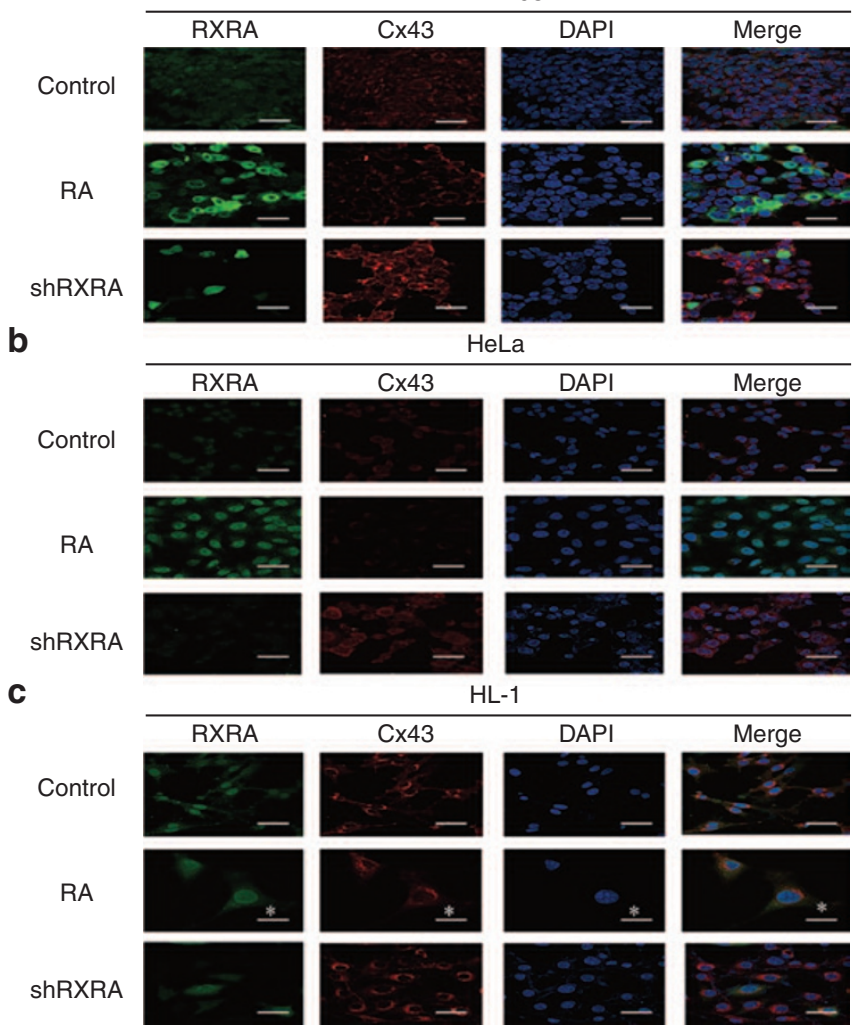

Figure 3. $R X R \alpha$ protein was localized to the nucleus and $C X 43$ was regulated by the level of $R X R \alpha$. Cells were treated with $10 \mu \mathrm{mol} / \mathrm{l} 9$-cis RA for $4 \mathrm{~h}$ or transfected with $\operatorname{sh} R X R \alpha$. Colocaliztion of $R X R \alpha$ and $C X 43$ was showed in three cells with a merged image. Green fluorescence indicates localization of $R X R \alpha$. Red fluorescence indicates $C \times 43$. Blue fluorescence indicates nuclei. The images were captured at $63 \times$ magnification or $2 \times$ zoom magnification using confocal microscopy. Scale bar represents 30 and $15 \mu \mathrm{m}\left({ }^{*}\right)$ showed at the right corner. (a) HEK293 cells; (b) HeLa cells; (c) HL-1 cells

luciferase activation was not changed in pGL3-mutC $x 43$-Luc with $\operatorname{sh} R X R \alpha(P=0.545$, HEK293; $P=0.631$, HeLa; $P=0.978$, HL-1; Figure 4, panel e, right).

To further localize the putative RARE, we constructed a series of luciferase vectors with truncate promoters to test whether progressively deleting parts of the $C \times 43$ promoter would abolish 9cRA-dependent transactivation. In the nonRA-treated group of three cell lines, the relative luciferase activation decreased significantly in -314-Cx43-Luc and -226-Cx43-Luc compared to that in -1426-Cx43-Luc (the same construction as PGL3-Cx43-Luc) (relative luciferase activation of -314-Cx43-Luc and -226-Cx43-Luc, respectively reduced to $\sim 2.84$-fold and $\sim 10.57$ in HEK293; 2.41 -fold and 5.61-fold in HeLa; 4.41- and 13.45-fold in HL-1; Figure 4, panel c). When $9 \mathrm{cRA}$ was added, the relative luciferase activation reduced only in -1426-Cx43-Luc $(P<0.001$, HEK293; $P<$ 0.001 , HeLa; $P<0.001$, HL-1; Figure 4, panel b, left); however, 9cRA did not affect the luciferase activation of -314-Cx43-Luc $(P=0.584$, HEK293; $P=0.053$, HeLa; $P=0.138$, HL-1; Figure 4, panel f, left) and -226-Cx43-Luc $(P=0.837$, HEK293; $P=$ 0.879 , HeLa; $P=0.638$, HL-1; Figure 4, panel f, right). Our observation suggests that RARE is likely to reside in the region from $-1,426$ to -314 base pairs upstream from the transcriptional start site.

\section{$R X R \alpha$ Interacts Directly With CX43 via RARE}

To confirm the interaction of $R X R \alpha$ with the RARE in the $C \times 43$ promoter, we performed an electrophoretic mobility shift assay (EMSA). A binding complex was formed between $R X R \alpha$ and the labeled wild-type $C x 43$ RARE with or without 9cRA (Figure 5, panel a, Lanes 1 and 2). However, this binding was not observed with the mutated probe (Figure 5, panel a, Lanes 3 and 4). When a cold RARE-probe was added in addition to the binding reaction, the binding was completely abolished (Figure 5, panel a, Lanes 5 and 6). However, the excess mutant RARE-probe failed to block this interaction (Figure 5, panel a, Lanes 7 and 8). Furthermore, we performed a shiftwestern assay to clarify the component of $R X R \alpha / \mathrm{RARE}$. The results of the shift-western assay showed that $R X R \alpha$ could bind to Cx43 RARE whether 9cRA existed or not (Figure 5, panel b). Collectively, these data indicate that $R X R \alpha$ specifically binds to the RARE in the $C \times 43$ gene promoter.

\section{The Identification of the $R X R \alpha$ Binding of $C x 43$ by ChIP Assay}

A chromatin immunoprecipitation assay (ChIP) was performed to investigate whether RXR $\alpha$ is able to bind to the $C \times 43$ promoter in vivo. Non-RA and 9cRA-treated HeLa cells were used in the ChIP assay. A specific PCR product was detected when the cells were treated with a specific anti-RXR $\alpha$ antibody, but were not detected when treated with a nonspecific immunoglobulin G control (Figure 5, panel c). DNA fragments containing Cx43 RARE sequences were detected in the PCR products (Figure 5, panel d). In quantitative real-time PCR, a significant enrichment of $R X R \alpha$ on the CX43 RARE was observed in both groups (the $\%$ input immunoprecipitated with the $R X R \alpha$ antibody and normal IgG was $\sim 2.5$ vs. $0.09 \%$, dimethylsulfoxide (DMSO) and 1.93 vs. $0.08 \%$, 9cRA group (Figure 5, panel e). Taken together, our findings indicate that $R X R \alpha$ could bind to the CX43 RARE directly.

\section{DISCUSSION}

Changing the level of $C x 43$ could induce heart defects $(7,8,26)$, and Cx43 might influence cardiac neural crest cell (CNCC) development and migration (27-29); CNCC is crucial for remodeling the vascular system, conotruncal outflow tract septation and the development of the cardiac conduction system (30). Consequently, the identification and understanding of the molecular signaling pathways that regulate $C \times 43$ function are important. In the present study, we demonstrated that 9cRA downregulates $C \times 43$ protein and mRNA levels and also demonstrated that this repression is a direct transcriptional effect that is mediated by the binding of $R X R \alpha$ to the $C x 43$ promoter.

The RA signal plays an essential role in embryogenesis, and numerous RA-response genes have been identified, only a few of which are unequivocally direct targets of the RA-receptor complex (16,31). The well-known RA target genes, such as $R A R \beta$, CYP26, CRABP-II and some Hox gene family members, 
a

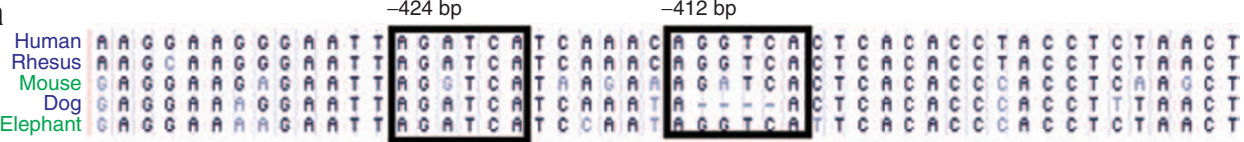

b AAGGAAGGGAATTAGATCATCAAACAGGTCACTCACACCTACCTCTAACT -WT AAGGAAGGGAATTAGCCCATCAAACAGGTCACTCACACCTACCTCTAACT - MT
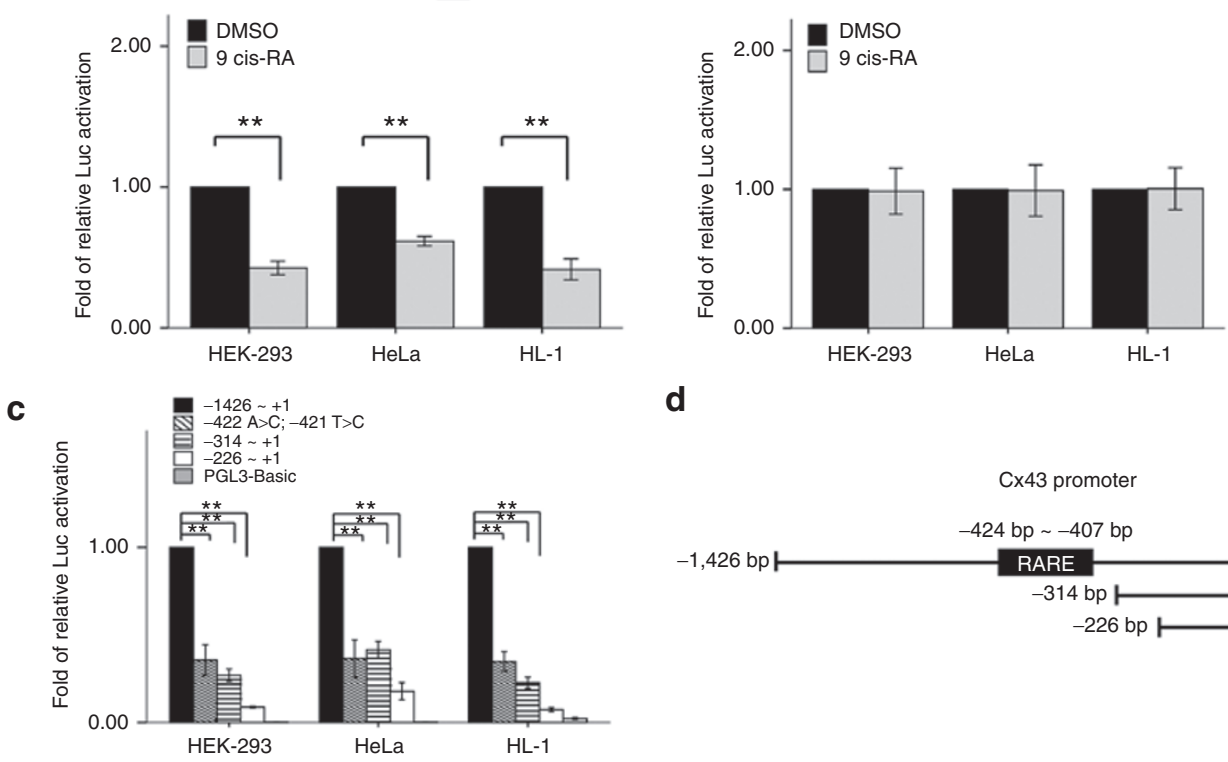

d

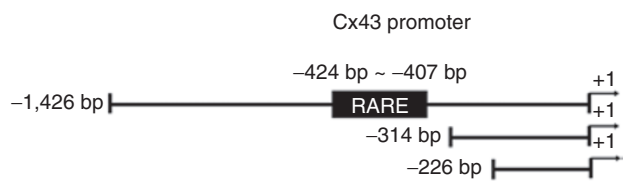

e
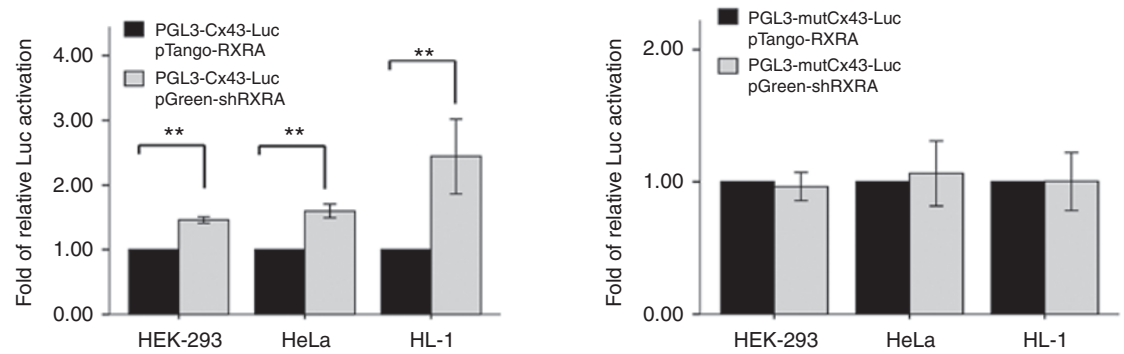

f
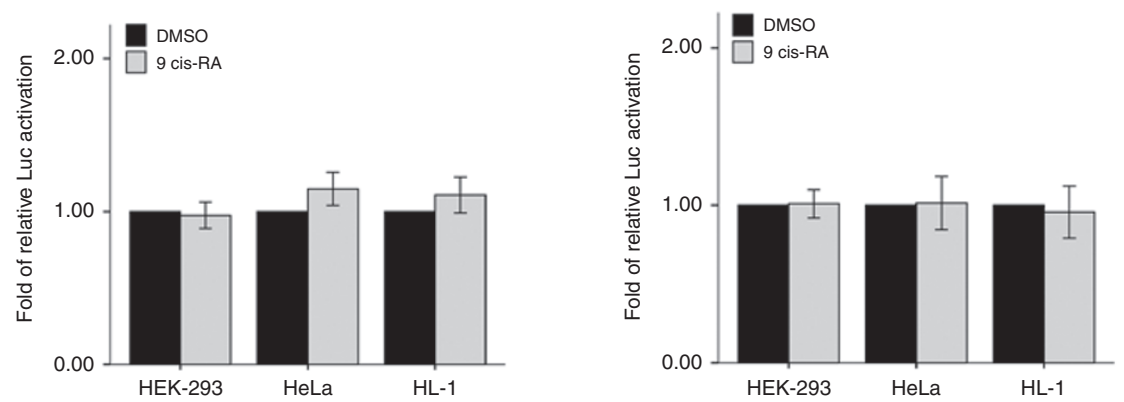

Figure 4. Liganded $R X R \alpha$ represses the activation of $C x 43$ promoter by a retinoic acid response element (RARE) from -424 to -407 base pairs. (a) The alignment of the human, mouse, rhesus, dog, and elephant $C x 43$ promoters. Sequence ranges from -438 to -389 base pairs in the $C x 43$ promoter, with a border outlining RARE. (b) wtRARE or mutRARE luciferase constructs were cotransfected with human RXR $\alpha$ expression vectors into HEK293, HeLa and $\mathrm{HL}-1$ cells, which were treated with DMSO or 9cRA. The luciferase activities were measured $48 \mathrm{~h}$ after transfection. left panel, wild-type constructs; right panel, mutRARE construct (c,f) HEK293, HeLa and HL-1 cells were cotransfected with human Cx43-luc truncation constructs, mutant Cx43-luc and human $R X R \alpha$ expression vectors were treated as described in $\mathrm{b}$, and the luciferase activities were analyzed. f, left panel, -314-Cx43-Luc; $\mathrm{f}$, right panel, -226-Cx43Luc (d) Schematic representation of the human $C x 43$ deletion constructs. The arrow indicates the transcription start site at +1 , and the RARE is labeled on the graph. (e) wtRARE- or mutRARE luciferase constructs were then co-transfected with pGreen-shRXR $\alpha$ into HEK293, HeLa and HL-1 cells, and the luciferase activities were measured after $24 \mathrm{~h}$. The mean values $( \pm)$ were calculated from three independent experiments. Bars, $S E$. ${ }^{*} P<0.05,{ }^{* *} P<0.001$.

are activated by RA-receptor complexes binding to RARE in their promoters $(32,33)$. In addition to its kinetic function, the RA-receptor complex also suppresses target genes, for example, $F g f 8$ and $T b x 2$, by binding to the RARE in their promoters
$(34,35)$. Our results showed that $R X R \alpha$ binds to a conserved RARE from -424 to -407 base pairs in the $C \times 43$ promoter to repress $C x 43$ expression and identify $C x 43$ as a new direct target of the RA signal. 
a

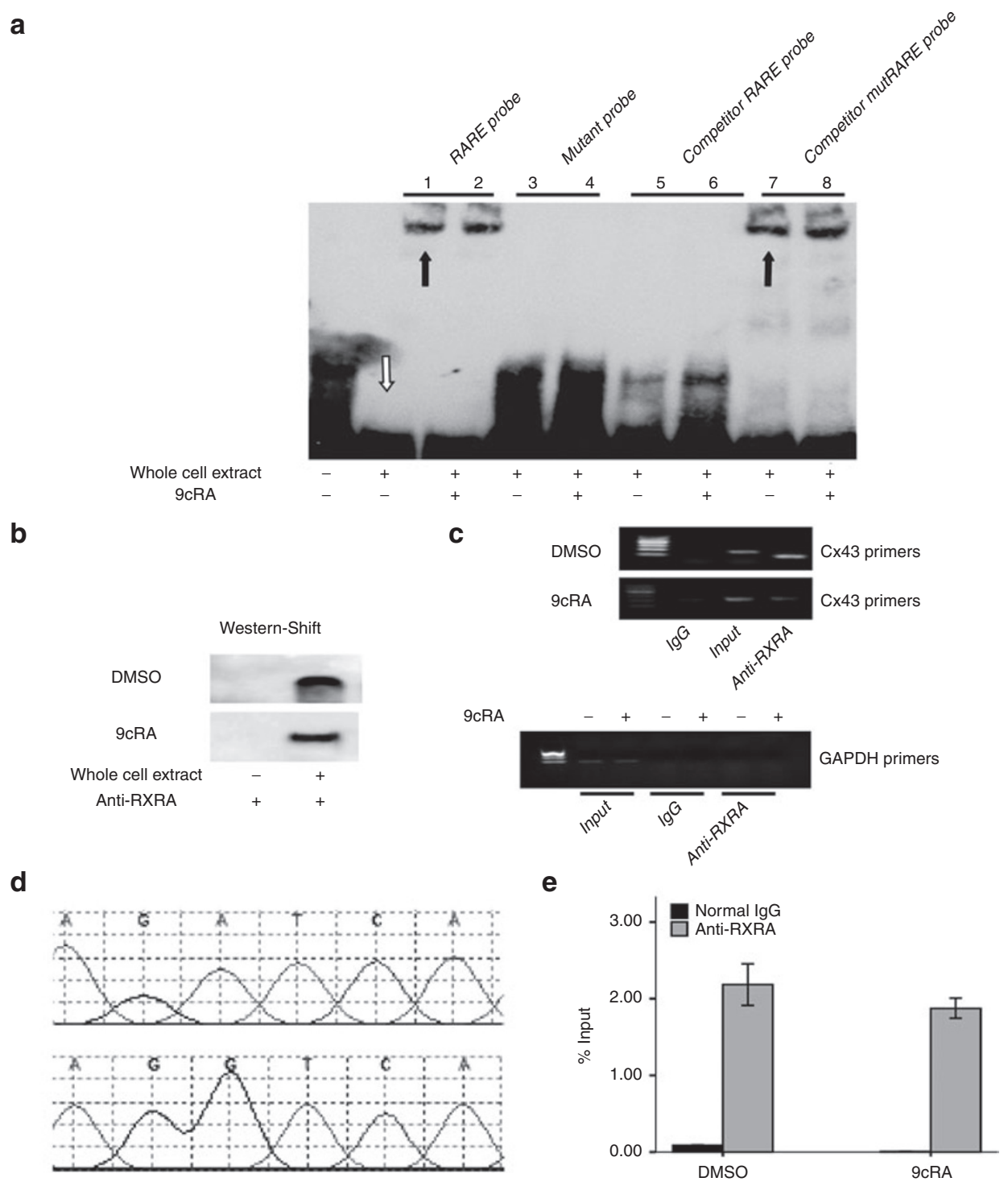

d

b

Figure 5. $R X R \alpha$ binds to $C X 43$ retinoic acid response element (RARE) directly. DMSO- or 9cRA-treated HeLa whole-cell extracts were incubated with biotinylated wild-type, mutant Cx43 RARE and unlabeled competitor probes in electrophoretic mobility shift assay (EMSA). (a) The left-most lane contains wild-type $C x 43$ RARE alone. The white arrow and the black arrow indicated the free probe and specific DNA-protein complex shift. Line 1, 2: wild-type probe + protein; Line 3,4: mutant probe + protein; Line 5, 6 unlabeled competitor wild-type probe + protein; Line 7, 8: unlabeled competitor mutant probe + protein (b) After EMSA, the DNA-protein complex was analyzed by western blotting with anti-RXR $\alpha$. (c,e) HeLa cells were treated with $10 \mu$ mol/l 9cRA or DMSO (control) for $24 \mathrm{~h}$, and chromatin immunoprecipitation assays were performed with an antibody against $R X R \alpha$ or lgG (negative control). Then, $10 \%$ input DNA and immunoprecipitated DNA were assayed by PCR and quantitative real-time polymerase chain reaction with $C x 43$ primers for the RARE. (d) The immunoprecipitated DNA fragments were sequenced after PCR. Bar, SE.

$R X R \alpha$, which is activated by 9 -cis retinoic acid (9cRA), can regulate cardiac gene expression $(23,36)$. In a previous study, the basal transcriptional activity of an atrial naturietic factor $(A N F)$ gene was repressed in the presence of 9cRA (37). Similar to our findings, $C \times 43$ was downregulated at both the protein and mRNA levels by increasing endogenous RXR $\alpha$ in HEK293, HeLa, and HL- 1 cells. Although RXR $\alpha$ did not increase significantly in high concentration of 9cRA $(100 \mu \mathrm{mol} / \mathrm{l}), C x 43$ still decreased while the ligand existed. We also knocked down RXR $\alpha$ expression in HEK293, HeLa and HL-1 cells to determine the role of $R X R \alpha$ in regulating $C x 43$. The results showed that $C \times 43$ expression increased whether 9cRA existed or not, indicating that $R X R \alpha$ played a crucial role in 9cRA suppressing $C \times 43$. Taken together, we conclude that liganded $R X R \alpha$ is criti$\mathrm{cal}$ for the observed repressive effect on $C \times 43$ gene expression.

Several studies have suggested that the target gene of the RA signal generally contains two direct-repeat half sites of the consensus sequence AGGTCA that are spaced by one to five base pairs $(14,16,32,38)$. RXR $\alpha$ homodimers activate transcription in response to $9 \mathrm{cRA}$ by binding to direct repeats that are spaced by one base pair (DR1 element) $(16,23,39)$. An analysis of the Cx43 promoter region revealed a novel putative RARE that is spaced by six base pairs ranging from $-1,426$ to +1 base pair upstream of the transcription start region. However, 
Table 1. Primer pairs of the $C x 43$ promoter sequence

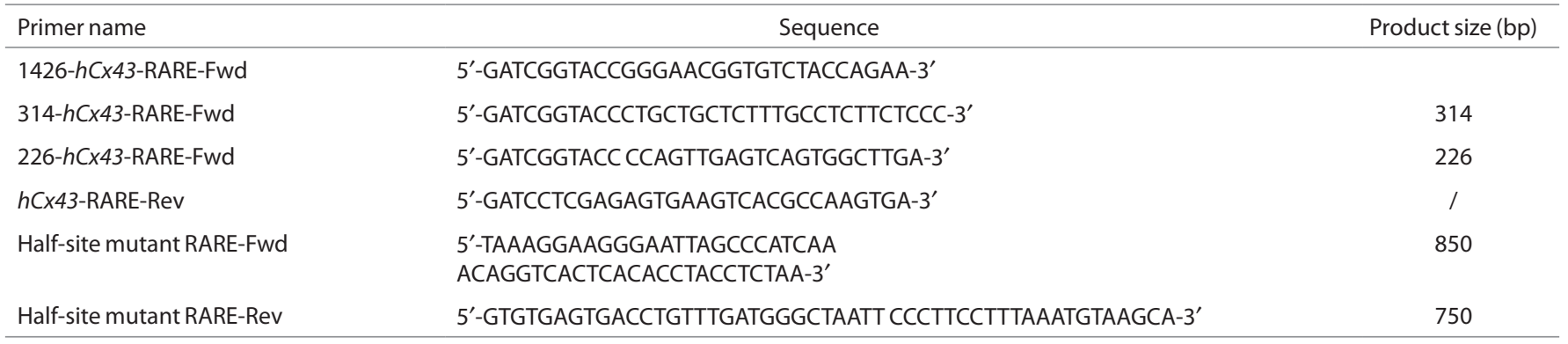

Table 2. Sequence of $\operatorname{sh} R X R \alpha$

\begin{tabular}{ll}
\hline shRNA & \multicolumn{1}{c}{ Sequence } \\
\hline Human-RXRa-sh $R X R \alpha$-sense & GATCCCAAGGACTGCCTGATTGACA ATTCAAGAGATTGTCAATCAGGCAGTCCTTGTTTTTG \\
Human-RXRa-sh $R X R \alpha$-antisense & AATTCAAAAACAAGGACTGCCTGA TTGACAATCTCTTGAATTGTCAATCAGGCAGTCCTTGG \\
Mouse-RXRa-shRXR $\alpha$-sense & GATCCCCTGTTCAACCCTGACTCTAATTCAAGAGATTAGAGTCAGGGTTGAACAGGTTTTTG \\
Mouse-RXRa-sh $R X R \alpha$ - anti-sense & AATTCAAAAACCTGTTCAACCCTGACTCTAATCTCTTGAATTAGAGTCAGGGTTGAACAGGG
\end{tabular}

Xavier-Neto's review demonstrated that the magic AGGTCA has high affinity but poor specificity (16). Some other NRs also utilized the RARE with the same spacer models that are used by RXRs/RARs, for example, orphan receptors, vitamin $D$ receptors $(V D R)$ and peroxisome proliferator-activated receptors (PPAR) $(32,39)$. Identifying a bona fide RARE is more difficult than a simple inspection. In order to attribute the RARE in Cx43 to a candidate sequence, some observations have been conducted in our study using molecular, biological and biophysical methods and functional approaches. In a ligand-dependent luciferase assay, RARE was located between the $-1,426$ to -341 base pair position. The constitutively active mutant Cx43 RARE represses the luciferase activity in the absence of the ligand and has no response to the $9 \mathrm{cRA}$. Our findings indicate that RARE in the Cx43 promoter is a functional element.

The HeLa cell line, with a high level of retinoic acid receptors (40), is an ideal cell line for the EMSA and ChIP assay. In the EMSA experiment, the proteins could interact with the $C \times 43$ RARE, and the retarded band was completed after excess unlabeled RARE probe was added. We also designed the labeled mutant probe in this assay, and these mutant probes failed to form a retardation band in the gel. A shift-western assay provided additional evidence that $R X R \alpha$ binds to RARE regardless of whether the cells were treated or not treated with 9cRA. In addition to EMSA, we conducted a ChIP assay to determine the relationship between $R X R \alpha$ and $C x 43$ in vivo. Consistent with the EMSA results, $C \times 43$ RARE could recruit $R X R \alpha$. These results suggest that $C \times 43$ RARE is a functional element in the RA signaling pathway and that liganded $R X R \alpha$ could repress Cx43 via this functional RARE.

Gene activation through RARE functions through the RA-dependent recruitment of transcriptional coactivators (41); however, the mechanism by which RAREs repress transcription is unclear. Kumar and colleagues (34) suggested an RA-dependent repressive role of the Fgf8 RARE by recruiting $P R C 2$ and the repressive H3K27me3 mark to the vicinity of the
Fgf8 RARE in trunk tissue. Whether 9cRA repressed Cx43 via RARE through this similar mechanism or another regulation pathway is unclear. Moreover, our study was limited at the cellular level, and the regulation pathway in heart tissue remains unknown. As a result, additional studies are needed to illustrate the regulation mechanism between RA and Cx43-RARE in animal models or patients.

In conclusion, the RARE-like sequence in the Cx43 promoter region may serve as a functional RARE in the RA signaling pathway. $R X R \alpha$ directly binds to this RARE and regulates the transcription and expression of the $C x 43$ gene.

\section{METHODS}

\section{Plasmid Construction}

Report assay. The human Cx43 (hCx43) (ENSG00000152661) promoter sequence spanning 1426 base pairs upstream of the translational start codon and serial truncations of the $\mathrm{h} C x 43$ gene promoter were produced by PCR amplification using the specific $5^{\prime}$ primers and the same 3' primer (Table 1). Then, these sequences were cloned into pGL3 basic (Promega, Madison, WI) using KpnI and XhoI sites to produce the -1426-Cx43-Luc, -314-Cx43-Luc and -226-Cx43-Luc vectors.

Site-directed mutagenesis of the hCx43 promoter. The left-half site of hCx43 RARE at positions -422 and -421 was mutated from AGATCA to AGCCCA using the primers in Table 1. The 1426-base pair hCx43luciferase plasmid with mutant RARE was produced by overlap PCR with the primer pairs mentioned above. The successful introduction of the mutation was confirmed by sequencing (Shanghai Invitrogen, Shanghai, China).

Express vector. The human pTango- $R X R \alpha$-Flag and pTango-sh $R X R \alpha$ expression plasmids were provided by Chengdu Lingdong Technology. The human and mouse pGreen-shRXRA (pGreen-human-shRXR $\alpha$ and pGreen-mouse-sh $R X R \alpha$ ) lentiviral plasmids were designed on the website (Sigma Life Science Biotools, St. Louis, MO) (Table 2) and cloned into pGreen (Promega) using BamHI and EcoRI sites.

\section{Cell Lines and Culture Conditions}

The human embryonic kidney cells (HEK 293 cells), HeLa cells and HL-1 cells were cultured in Dulbecco's modified Eagle medium (Hyclone, Logan, UT) that was supplemented with $10 \%$ fetal bovine serum (Gibco BRL, Langley, OK) and penicillin $(100 \mathrm{U} / \mathrm{ml})$-streptomycin $(100 \mu \mathrm{g} / \mathrm{ml})$ and maintained at $37^{\circ} \mathrm{C}$ in a $5 \% \mathrm{CO}_{2}$ atmosphere. 


\section{9-cis RA Treatment}

For western blotting and quantitative real-time PCR, HEK293 cells, HeLa cells and HL-1 cells were treated with $0,1,10$, and $100 \mu \mathrm{mol} / \mathrm{l}$ 9 -cis retinoic acid (Sigma-Aldrich) to upregulate endogenous $R X R \alpha$. The HEK293 cells and HeLa cells were transfected with pGreenhuman-shRXR $\alpha$ to downregulate $R X R \alpha$ expression. These cells were also treated with 9-cis RA for $24 \mathrm{~h}$. For EMSA, HeLa cells were plated in $10-\mathrm{cm}$ dishes and treated with $10 \mu \mathrm{mol} / 1$ 9cRA for $24 \mathrm{~h}$. The control groups were treated by the same concentration of DMSO (Sigma-Aldrich).

\section{Lentiviral Stable Knockdown HL-1 Cells}

HEK293 cells at $\sim 70 \%$ confluency in a 12 -well plate were transfected using Lipofectamine 2000 (Invitrogen Life Technologies, Carlsbad, CA) with $400 \mathrm{ng}$ of the pGreen-mouse-sh $R X R \alpha$ lentiviral vector and $400 \mathrm{ng}$ of a mixture of Gag-Pol packaging plasmid (pRRE), Rev packaging plasmid (PRSV-Rev), vesicular somatitis virus $G$ envelope plasmid (pVSVG) per well. The virus supernatants were harvested 24 and $48 \mathrm{~h}$ after transfection. HL-1 cells were transduced with viral particles in the presence of $5 \mu \mathrm{g} / \mathrm{ml}$ polybrene (hexadimethrine bromide; Sigma-Aldrich) overnight. A pure population of positively transduced cells was selected and enriched by applying puromycin in culture medium. The successful knockdown of $R X R \alpha$ in the $\operatorname{sh} R X R \alpha$-HL-1 cells was confirmed by western blotting and qRT-PCR.

\section{Immunofluorescence}

The cells were grown on coverslip slides, transfected with $800 \mathrm{ng}$ of the pTango-sh $R X R \alpha$ plasmids or treated with $10 \mu \mathrm{mol} / 1$ 9-cis RA for $4 \mathrm{~h}$. The cells were washed with phosphate buffered saline and fixed with $4 \%$ paraformaldehyde before permeabilization with $0.3 \%$ Triton X-100. The slides were incubated overnight at $4{ }^{\circ} \mathrm{C}$ with rabbit $R X R \alpha$ monoclonal antibody (ab125001; Abcam, Cambridge, UK) and mouse $C \times 43$ monoclonal antibody (sc-271837; Santa Cruz Biotechnology, Dallas, TX) at a dilution of 1:150. The next day, the cells were incubated with Fluorescein (FITC) goat anti-rabbit IgG (Jackson ImmunoResearch, West Grove, PA) and Alexa Fluor 594 goat anti-mouse IgG (Jackson ImmunoResearch) at a dilution of 1:200. The cells were incubated with $1 \mu \mathrm{g} / \mathrm{ml}$ DAPI (4, 6-diamidino2-phenylindole, Sigma-Aldrich), mounted on a slide and examined by confocal fluorescence microscopy.

\section{Western Blotting}

The cells were harvested and solubilized in radio immunoprecipitation assay lysis buffer $(50 \mathrm{mmol} / \mathrm{l}$ Tris- $\mathrm{HCl}, 150 \mathrm{mmol} / \mathrm{l} \mathrm{NaCl}, 1 \% \mathrm{NP}-40$, $0.1 \%$ sodium dodecyl sulfonate) containing protein inhibitor cocktail (Roche, Basel, Switzerland) and phosphate inhibitor (Roche) on ice. The protein concentrations were determined using the bicinchoninic acid assay (Pierce, Rockford, IL) according to the manufacturer's instructions, with bovine serum albumin as the standard. Then, $10 \mu \mathrm{g}$ of protein was loaded onto and separated in $10 \%$ sodium dodecyl sulfonate-polyacrylamide gels. Nitrocellulose membranes (Whatman, GE Healthcare Life Sciences, Buckinghamshire, UK) were blocked for $1 \mathrm{~h}$ at room temperature with phosphate-buffered saline containing 10\% nonfat milk. Appropriate primary antibodies were incubated with the membranes, followed by peroxidase-conjugated anti-rabbit antibody (dilution at 1:5,000; Pierce), and visualized by enhanced chemiluminescence (Pierce). The primary antibodies that were used were rabbit polyclonal anti-Cx43 (sc-9059; Santa Cruz Biotechnology) and rabbit monoclonal anti-RXR $\alpha$ (ab125001; Abcam). Anti-GAPDH (21612; Signalway Antibody, Park, MD) was used as a loading control.

\section{RNA Extraction and Quantitative Real-Time PCR}

The total RNA was extracted from the cells using the Quick-RNA MicroPrep kit (Zymo Research, Irvine, CA). For each sample, $1 \mu \mathrm{g}$ of total RNA was used for cDNA synthesis with the PrimeScript RT reagent Kit (Takara Biotechnology, Dalian, China) in a $20-\mu \mathrm{l}$ reaction mix in accordance with the manufacturer's instructions. The final cDNA product was diluted 10 -fold and subsequently used as a template for real-time PCR. Amplification reactions were carried out using the SYBR Premix Ex Taq Kit (Takara Biotechnology) and were performed in triplicate in 384-well-plate format using an Applied Biosystems 7900HT Fast Real-time PCR System (Applied Biosystems,
Table 3. Primers for real-time polymerase chain reaction

\begin{tabular}{|c|c|c|}
\hline Primer name & Sequence & $\begin{array}{l}\text { Product } \\
\text { size (bp) }\end{array}$ \\
\hline $\mathrm{h} R X R \alpha$-Fwd & 5'-GCAAGGACCTGACCTACACC-3' & 171 \\
\hline $\mathrm{h} R X R \alpha-\operatorname{Rev}$ & 5'-CACCTCATTCTCGTTCCGGT-3' & \\
\hline $\mathrm{m} R X R \alpha-\mathrm{Fwd}$ & 5'-GTCCGCCCTTCTCTGTCATC-3' & 175 \\
\hline $\mathrm{m} R X R \alpha-\operatorname{Rev}$ & 5'-GGAACCTTGAGGACGCCATT-3' & \\
\hline hCx43-Fwd & $5^{\prime}$-CAGCTTGTACCCAGGAGGAG-3' & 209 \\
\hline hCx43-Rev & 5'-TGTCCCTGGCCTTGAATATC-3' & \\
\hline $\mathrm{mCx} 43-\mathrm{Fwd}$ & $5^{\prime}$-CAGCGCAGAGCAAAATCGAA-3' & 156 \\
\hline $\mathrm{mCx} 43-\operatorname{Rev}$ & $5^{\prime}$-CTGGAAGGTCGCTGATCCAC-3' & \\
\hline hBeta-actin-Fwd & 5'-CCTTCCTGGGCATGGAGTCCT-3' & 150 \\
\hline hBeta-actin-Rev & 5'-AATCTCATCTTGTTTTCTGCG-3' & \\
\hline mBeta-actin-Fwd & 5'-CACACCCGCCACCAGTTCG-3' & 151 \\
\hline mBeta-actin-Rev & 5'-GTCCTTCTGACCCATTCCCACC-3' & \\
\hline
\end{tabular}

Foster City, CA). The relative quantification of gene expression was calculated using the $2^{-\Delta \Delta} \mathrm{Ct}$ method. The $\beta$-actin expression levels were used for the normalization of gene expression values. The primers of $R X R \alpha$ and $C \times 43$ for real-time PCR are listed in Table 3.

\section{Transfection and Luciferase Assay}

Transient transactions cells were plated in 48 -well plates $24 \mathrm{~h}$ before transfection and performed using Lipofectamine 2000 (Invitrogen Life Technologies) and X-tremeGENE HP DNA Transfection Reagent (Roche) according to the manufacturer's instructions. We cotransfected cells with $500 \mathrm{ng}$ of the pGL3-Basic, -1426-Cx43-Luc (pGL3-Cx43-Luc), -1426-Cx43-mutRARE (pGL3-mutCx43-Luc), -314-Cx43-Luc, -226-Cx43-Luc and $R X R \alpha$ expression plasmids or pGreen-sh $R X R \alpha$ overnight. The next day, the medium was replaced with fresh medium containing $10 \mu \mathrm{mol} / \mathrm{l} 9 \mathrm{cRA}$ and incubated for another $24 \mathrm{~h}$. The cells extracts were used for the luciferase activities measurement by the dual luciferase assay system (Promega). pRLRenilla (Promega) was used as a normalized plasmid.

\section{EMSA}

Whole HeLa cell extracts were treated with DMSO (control) or 10 $\mu \mathrm{mol} / \mathrm{l}$ 9cRA. The concentration of these proteins was measured by the bicinchoninic acid assay (Pierce) using bovine serum albumin as a standard. Biotin-labeled double-stranded oligonucleotide probes containing wild-type and mutant Cx43 RARE sequences were bound to whole-cell extracts. The binding reactions were performed using a Light Shift Chemiluminescent EMSA kit (Pierce) according to the manufacturer's protocols. Then, 1 pmol of biotin-labeled duplex oligonucleotides bearing either the RARE or mutRARE was incubated with $10 \mu \mathrm{g}$ of whole-cell extracts for $10 \mathrm{~min}$ in $10 \times$ binding buffer mixed with $1 \mu \mathrm{g} / \mu \mathrm{l}$ poly $(\mathrm{dI} \bullet \mathrm{dC}), 50 \%$ glycerol and $1 \% \mathrm{NP}-40$. In competition studies, 200- to 400-fold molar excesses of unlabeled competitor oligonucleotides was added to the reaction. Then, the reaction mixture was electrophoresed on a native $6 \%$ polyacrylamide gel and transferred to a positive nylon membrane (Merck Millipore, Darmstadt, Germany). The membrane was optimally UV-light cross-linked, and detection was performed using stabilized streptavidin-horseradish peroxidase conjugate (Pierce) and photographed by the Fuji film Las 3000 Luminescent Image Analyzer (Fuji Life Sciences, Tokyo, Japan). The sequences of synthetic duplex oligonucleotide probes are in Table 4.

\section{Shift-Western Blotting}

The combination of the gel shift and the protein immunoblot (called "shift-western blotting") identifies the individual components of protein-DNA complexes (42). Western blotting was performed after EMSA. For transfers, stacked membranes were used: the first filter below the gel was a nitrocellulose membrane (Whatman), followed by a nylon membrane (Merck Millipore). All of the filters were wetted 
Table 4. Electrophoretic mobility shift assay 5'-biotin-labeled oligos and unlabeled oligos

\begin{tabular}{ll}
\hline Oligonucleotides name & \multicolumn{1}{c}{ Sequence } \\
\hline Biotin-Cx43-RARE-Fwd & 5'-AATTAGATCATCAAACAGGTCACTCA-3' \\
Biotin-Cx43-RARE-Rev & 5'-TGAGTGACCTGTTTGATGATCTAATT-3' \\
Biotin-mutCx43-RARE-Fwd & 5'-AATTAGCCCATCAAACAGGTCACTCA-3' \\
Biotin-mutCx43-RARE-Rev & 5'-TGAGTGACCTGTTTGATGGGCTAATT-3' \\
\hline
\end{tabular}

Table 5. Chromatin immunoprecipitation oligos

\begin{tabular}{llc}
\hline Primer name & \multicolumn{1}{c}{ Sequence } & $\begin{array}{c}\text { Product } \\
\text { size }(\mathrm{bp})\end{array}$ \\
\hline ChIP- $h C x 43-$ Fwd & 5'-CTCTCCCATCTCCACCATTCC-3' $^{\prime}$ & 155 \\
ChIP-hCx43-Rev & $5^{\prime}$-TGGTGGGGAAGCATATAACCA-3' & \\
ChIP-GAPDH-Fwd & 5'-TACTAGCGGTTTTACGGGCG-3' $^{\prime}$ & 160 \\
ChIP-GAPDH-Rev & 5'-TTCGAACAGGAGGAGCAGAGAGCGA-3' $^{\prime}$ & \\
\hline
\end{tabular}

in $0.5 \times$ Tris-Borate-EDTA; all of the filter papers (Whatman $3 \mathrm{MM}$ ) and towels were equilibrated in the appropriate transfer buffer. The radioactivity on nylon membranes was measured as EMSA. For protein detection, the membranes were first blocked for at least $45 \mathrm{~min}$ with phosphate-buffered saline containing $10 \%$ nonfat milk powder. Rabbit monoclonal anti-RXR $\alpha$ (ab125001; Abcam) was incubated with the membrane at a dilution of 1:1,000, followed by peroxidaseconjugated anti-rabbit antibody $(1: 5,000)$, and visualized by enhanced chemiluminescence (Pierce).

\section{ChIP}

HeLa cells were plated in $10-\mathrm{cm}$ dishes at a total of $2 \times 10^{7}$ cells and treated with or without $10 \mu \mathrm{mol} / \mathrm{l} 9 \mathrm{cRA}$ for $24 \mathrm{~h}$. The cells were then cross-linked with $1 \%$ formaldehyde for $10 \mathrm{~min}$. Immunoprecipitation was performed using a Pierce Agarose ChIP Kit (Pierce). The chromatin fragments of 200-400 bp were incubated with $6 \mu \mathrm{g}$ of rabbit polyclonal antibody anti- $R X R \alpha$ (sc-553X; Santa Cruz Biotechnology) and $2 \mu \mathrm{g}$ of nonspecific IgG that was provided in the kit at $4{ }^{\circ} \mathrm{C}$ overnight. Ten percent of supernatant volume was reserved as input. The identity and quantity of the DNA fragments were determined by PCR and quantitative real-time PCR. The PCR products were visualized after electrophoresis on a $1.5 \%$ agarose gel. The primer pairs that were used for PCR and quantitative real-time PCR are shown in Table 5.

\section{Statistical Analysis}

Each experiment in this study was repeated at least three times. The data were analyzed using SPSS 17.0 (International Business Machines Corporation, Armonk, NY). Significant differences were determined by ANOVA with a significance level of $P<0.05$. The qRT-PCR and luciferase assay were presented as the mean \pm SE.

\section{Statement From our Institutional Review Board}

The study had been approved by Institutinal Review Board of Children's Hospital of Fudan University. And we had not used any clinical samples in our study not use any clinical sample in our study.

\section{CREDIT FOR AUTHORSHIP}

Conception and study design: G.H., X.M., R.G., J.Z., J.X., and Y.L. Data analysis: R.G. Drafting: R.G. and W.S. Final approval: R.G., J.X., Y.L., J.Z., H.W., D.M., X.M., and G.H.

\section{STATEMENT OF FINANCIAL SUPPORT}

This work was supported by grants from the National Basic Research Program of China (2010CB529504), the National Natural Science Foundation of China (30930096, 81570282, 81570283), and Shanghai Municipal Commission of Science and Technology Research Projects (11JC1401400).

Disclosure: The authors declare no competing interests.

\section{REFERENCES}

1. Zhao QM, Ma XJ, Ge XL, et al.; Neonatal Congenital Heart Disease screening group. Pulse oximetry with clinical assessment to screen for congenital heart disease in neonates in China: a prospective study. Lancet 2014;384:747-54.

2. Hoffman Jle. The global burden of congenital heart disease. Cardiovasc J Afr 2013;24:141-5.

3. Kathiriya IS, Nora EP, Bruneau BG. Investigating the transcriptional control of cardiovascular development. Circ Res 2015;116:700-14.

4. Nemer M. Genetic insights into normal and abnormal heart development. Cardiovasc Pathol 2008; 17:48-54.

5. Salat-Canela C, Sesé M, Peula C, Ramón y Cajal S, Aasen T. Internal translation of the connexin 43 transcript. Cell Commun Signal 2014;12:31.

6. Jeyaraman MM, Srisakuldee W, Nickel BE, Kardami E. Connexin43 phosphorylation and cytoprotection in the heart. Biochim Biophys Acta 2012;1818:2009-13.

7. Huang GY, Wessels A, Smith BR, Linask KK, Ewart JL, Lo CW. Alteration in connexin 43 gap junction gene dosage impairs conotruncal heart development. Dev Biol 1998;198:32-44.

8. Ewart JL, Cohen MF, Meyer RA, et al. Heart and neural tube defects in transgenic mice overexpressing the $\mathrm{Cx} 43$ gap junction gene. Development 1997;124:1281-92.

9. Britz-Cunningham SH, Shah MM, Zuppan CW, Fletcher WH. Mutations of the Connexin43 gap-junction gene in patients with heart malformations and defects of laterality. N Engl J Med 1995;332:1323-9.

10. Salameh A, Haunschild J, Bräuchle P, et al. On the role of the gap junction protein Cx43 (GJA1) in human cardiac malformations with Fallot-pathology. a study on paediatric cardiac specimen. PLoS One 2014;9:e95344.

11. Huang GY, Xie LJ, Linask KL, et al. Evaluating the role of connexin 43 in congenital heart disease: Screening for mutations in patients with outflow tract anomalies and the analysis of knock-in mouse models. J Cardiovasc Dis Res 2011;2:206-12.

12. Debrus S, Tuffery S, Matsuoka R, et al. Lack of evidence for connexin 43 gene mutations in human autosomal recessive lateralization defects. J Mol Cell Cardiol 1997;29:1423-31.

13. D'Aniello E, Waxman JS. Input overload: Contributions of retinoic acid signaling feedback mechanisms to heart development and teratogenesis. Dev Dyn 2015;244:513-23.

14. Mendoza-Parra MA, Gronemeyer H. Integrative genomics to dissect retinoid functions. Subcell Biochem 2014;70:181-202.

15. Piskunov A, Al Tanoury Z, Rochette-Egly C. Nuclear and extra-nuclear effects of retinoid acid receptors: how they are interconnected. Subcell Biochem 2014;70:103-27.

16. Xavier-Neto J, Sousa Costa ÂM, Figueira AC, et al. Signaling through retinoic acid receptors in cardiac development: Doing the right things at the right times. Biochim Biophys Acta 2015;1849:94-111.

17. Yang Y, Qin SK, Wu Q, et al. Connexin-dependent gap junction enhancement is involved in the synergistic effect of sorafenib and all-trans retinoic acid on HCC growth inhibition. Oncol Rep 2014;31:540-50.

18. Wu J, Taylor RN, Sidell N. Retinoic acid regulates gap junction intercellular communication in human endometrial stromal cells through modulation of the phosphorylation status of connexin 43. J Cell Physiol 2013;228:90310 .

19. Wang J, Dai Y, Huang Y, et al. All-trans retinoic acid restores gap junctional intercellular communication between oral cancer cells with upregulation of Cx32 and Cx43 expressions in vitro. Med Oral Patol Oral Cir Bucal 2013;18:e569-77.

20. van Veen TA, van Rijen HV, Wiegerinck RF, et al. Remodeling of gap junctions in mouse hearts hypertrophied by forced retinoic acid signaling. J Mol Cell Cardiol 2002;34:1411-23.

21. Zhang J, Ma X, Wang H, Ma D, Huang G. Elevated methylation of the RXRA promoter region may be responsible for its downregulated expression in the myocardium of patients with TOF. Pediatr Res 2014;75:588-94.

22. Wu Y, Ma XJ, Wang HJ, et al. Expression of Cx43-related microRNAs in patients with tetralogy of Fallot. World J Pediatr 2014;10:138-44.

23. Gilardi F, Desvergne B. RXRs: collegial partners. Subcell Biochem 2014;70:75-102. 


\section{Articles | Guetal.}

24. Wolf G. Is 9-cis-retinoic acid the endogenous ligand for the retinoic acid-X receptor? Nutr Rev 2006;64:532-8.

25. http://genome.ucsc.edu/.

26. Lyon RC, Mezzano V, Wright AT, et al. Connexin defects underlie arrhythmogenic right ventricular cardiomyopathy in a novel mouse model. Hum Mol Genet 2014;23:1134-50.

27. Xu X, Francis R, Wei CJ, Linask KL, Lo CW. Connexin 43-mediated modulation of polarized cell movement and the directional migration of cardiac neural crest cells. Development 2006;133:3629-39.

28. Lo CW, Waldo KL, Kirby ML. Gap junction communication and the modulation of cardiac neural crest cells. Trends Cardiovasc Med 1999;9:63-9.

29. Huang GY, Cooper ES, Waldo K, Kirby ML, Gilula NB, Lo CW. Gap junction-mediated cell-cell communication modulates mouse neural crest migration. J Cell Biol 1998;143:1725-34.

30. Keyte A, Hutson MR. The neural crest in cardiac congenital anomalies. Differentiation 2012;84:25-40.

31. Balmer JE, Blomhoff R. Gene expression regulation by retinoic acid. J Lipid Res 2002;43:1773-808.

32. Al Tanoury Z, Piskunov A, Rochette-Egly C. Vitamin A and retinoid signaling: genomic and nongenomic effects. J Lipid Res 2013;54:1761-75.

33. Gillespie RF, Gudas LJ. Retinoid regulated association of transcriptional co-regulators and the polycomb group protein SUZ12 with the retinoic acid response elements of Hoxa1, RARbeta(2), and Cyp26A1 in F9 embryonal carcinoma cells. J Mol Biol 2007;372:298-316.

34. Kumar S, Duester G. Retinoic acid controls body axis extension by directly repressing Fgf8 transcription. Development 2014;141:2972-7.
35. Sakabe M, Kokubo H, Nakajima Y, Saga Y. Ectopic retinoic acid signaling affects outflow tract cushion development through suppression of the myocardial Tbx2-Tgf $\beta 2$ pathway. Development 2012;139:385-95.

36. Minucci S, Leid M, Toyama R, et al. Retinoid X receptor (RXR) within the RXR-retinoic acid receptor heterodimer binds its ligand and enhances retinoid-dependent gene expression. Mol Cell Biol 1997;17:644-55.

37. Clabby ML, Robison TA, Quigley HF, Wilson DB, Kelly DP. Retinoid X receptor alpha represses GATA-4-mediated transcription via a retinoiddependent interaction with the cardiac-enriched repressor FOG-2. J Biol Chem 2003;278:5760-7.

38. Brazda P, Krieger J, Daniel B, et al. Ligand binding shifts highly mobile retinoid X receptor to the chromatin-bound state in a coactivator-dependent manner, as revealed by single-cell imaging. Mol Cell Biol 2014;34: 1234-45.

39. Moutier E, Ye T, Choukrallah MA, et al. Retinoic acid receptors recognize the mouse genome through binding elements with diverse spacing and topology. J Biol Chem 2012;287:26328-41.

40. Myga-Nowak M, Pacholska-Bogalska J, Kwaśniewski W, Kwaśniewska A, Goździcka-Józefiak A. Proliferation of cells and expression of RARs, RXRs and HPV viral E6 and E7 proteins in cervical cancer cell lines after treatment with ATRA. Ann Agric Environ Med 2011;18:145-50.

41. Germain P, Iyer J, Zechel C, Gronemeyer H. Co-regulator recruitment and the mechanism of retinoic acid receptor synergy. Nature 2002;415:187-92.

42. Demczuk S, Harbers M, Vennström B. Identification and analysis of all components of a gel retardation assay by combination with immunoblotting. Proc Natl Acad Sci USA 1993;90:2574-8. 